\title{
Change and Identity in Complex Systems
}

\author{
Graeme S. Cumming $^{1}$ and $\underline{\text { John Collier }}^{2}$
}

\begin{abstract}
Complex systems are dynamic and may show high levels of variability in both space and time. It is often difficult to decide on what constitutes a given complex system, i.e., where system boundaries should be set, and what amounts to substantial change within the system. We discuss two central themes: the nature of system definitions and their ability to cope with change, and the importance of system definitions for the mental metamodels that we use to describe and order ideas about system change. Systems can only be considered as single study units if they retain their identity. Previous system definitions have largely ignored the need for both spatial and temporal continuity as essential attributes of identity. After considering the philosophical issues surrounding identity and system definitions, we examine their application to modeling studies. We outline a set of five alternative metamodels that capture a range of the basic dynamics of complex systems. Although Holling's adaptive cycle is a compelling and widely applicable metamodel that fits many complex systems, there are systems that do not necessarily follow the adaptive cycle. We propose that more careful consideration of system definitions and alternative metamodels for complex systems will lead to greater conceptual clarity in the field and, ultimately, to more rigorous research.
\end{abstract}

Key Words: complexity; resilience; identity; adaptive cycle; limitation; replacement; random walk; evolution; ecosystem; economy; society; social-ecological system; metamodels

\section{INTRODUCTION}

This paper is concerned with the problem of how we can capture the dynamic, changing nature of complex systems, including ecosystems, social systems, and economies, in a cohesive conceptual framework. We discuss two central themes: the nature of system definitions and their ability to cope with change, and the mental metamodels that we use to describe and order ideas about system change. We argue in both instances that the current conceptual framework for studies of complex systems can only benefit from a more complete, explicit consideration of the themes of dynamic change and system identity.

\section{SYSTEM DEFINITIONS AND SYSTEM CHANGE}

An important step in research is to define the study system, or at the least to set bounds on the phenomena that are considered to be of interest. This limits the mechanistic scope of the study to focus on the most important aspects of the problem. Shifting study system definitions can create confusion and errors, particularly when different researchers are working on the same problem. A subjective definition that includes system limits and a scale or scales of interest must be imposed on the real world before we can achieve the clarity and rigor that good research demands (Sagoff 2003).

Despite the obvious importance of knowing what the object of research is, it can be difficult to find clear definitions of complex systems. Ecologists have for many years used the definition of Tansley (1935) for ecosystems, although the original 
definition has been modified through the years (e. g., Odum 1971, Likens 1992, O'Neill 2001, Pickett and Cadenasso 2002), and there is less consensus in sociology and economics. Discussions of change in complex systems often ignore the question of how to maintain a constant definition of a changing system. As we argue in the second half of this paper, system definitions have particular importance when developing models of system function. In some instances, we will need to distinguish between systems that maintain their identity over time and systems that effectively develop into new systems or adopt an entirely new identity. This level of distinction is not possible without a clear, unambiguous definition of what constitutes the system in the first place.

A useful metaphor for thinking about change and identity comes from the ancient philosophical problem of Theseus' ship. According to the Greek legend, Theseus slew the Minotaur and returned to Greece with the youth of Athens. On their return to Greece, it was decided to preserve Theseus' ship in perpetuity. The ship was moored and carefully maintained. Over the years, rotting ropes, timbers, and sails were gradually replaced with new ones. Plutarch (Vita Thesei, 75 A.D.; see Clough 1992) records it thus: "The ship wherein Theseus and the youth of Athens returned had thirty oars, and was preserved by the Athenians down even to the time of Demetrius Phalereus [ 350-280 B.C.], for they took away the old planks as they decayed, putting in new and stronger timber in their place, insomuch that this ship became a standing example among the philosophers, for the logical question of things that grow; one side holding that the ship remained the same, and the other contending that it was not the same." The question has now been debated for more than 2000 yr: was the ship still Theseus' ship?

If Thesus's ship were defined as an entity composed of the actual boards and sails that Theseus used, then the ship that lay in harbor gradually became less and less Theseus' ship. Alternatively, if the identity of the ship lay in its size, shape, number of sails, and so forth, then it remained that of Thesus. Between these two extremes lie numerous possible thought experiments. What if workmen a hundred years later had introduced a plank of a different timber, or painted the sails a different color? How many minor changes would the ship have been able to undergo while still maintaining its identity as Theseus'? If it sailed from the harbor under a different master, would it still be Theseus' ship? And if it stopped being Theseus' ship, at what point would it do so? Finally, imagine that the old materials had gradually been reassembled into the form of the original ship as they were removed. At the point when the original had been entirely reconstituted, would there be two ships of Theseus? These questions illustrate the ambiguity of the concept of identity over time. Spatial identity is similarly important, and, although it may be fairly straightforward for ships, it can be an issue for nation states and for many other kinds of complex systems.

The role played by our subjective interest in the system is in many ways crucial to our system definition. If we ask different questions about the system that we are studying, we can expect different answers, and, for the same question, the answer might depend on our motivations for asking it. For example, if we are mostly concerned with ownership, the same ship would not be Theseus' ship if it had a new owner. If we are concerned with the materials from which the ship is made, then Theseus' ship might be a pile of materials in a stockyard. If we are concerned with functionality, then Theseus' ship would be the same functional unit, irrespective of its material embodiment. Or perhaps there are legal criteria for ship identity, as for automobiles, and the ship is the same if its frame, with identification number, is intact, regardless of its functionality. Although legal identity is determined by a set of social conventions, such conventional criteria are not appropriate for many complex systems. Unlike the ship, which is an artifact, the systems that we are most concerned with are natural systems and must have natural identity conditions. The challenge of determining system identity is to establish the natural properties of our study systems that constitute identity conditions over time and space.

For ecosystems, which are more clearly defined than social or economic systems, Tansley (1935) defined the ecosystem as " ... the fundamental concept appropriate to the biome considered together with all the effective inorganic factors of its environment." More recently, Pickett and Cadenasso (2002) have argued that " ... the main components of the [ecosystem] concept are its abiotic and biotic features and the interactions between them." They add that, although the definition of ecosystems is independent of scale, "... all instances of ecosystems have an explicit spatial extent." As a result, Pickett and Cadenasso (2002) consider Theseus' ship to be defined by its materials, 
the relationships between them, and the location of the ship in the harbor.

Although this definition is a good starting point, there are circumstances under which it may be inappropriate or ambiguous. Resolution of the problems in the ecosystem concept is of particular importance when developing dynamic models of ecosystems. For instance, how important is the requirement for a spatially explicit definition, and must it be static? As the global climate warms, we can expect to see a shifting of the spatial boundaries of ecosystems. If the boundaries of a deciduous forest gradually change until they lie $50 \mathrm{~km}$ to the north of its original location, does it remain the same ecosystem? Many ecologists would say that it does, but the "explicit spatial extent" has changed. Alternatively, imagine a situation in which a large disturbance destroys the entire flora and fauna of a subcatchment. Recolonization from neighboring areas occurs, and a community develops that has exactly the same species composition and ecological functions as the previous one. Is the new ecosystem the same, or different? Many ecologists would consider that the new ecosystem, although it might be the same in all respects, is different. If we accidentally sink Theseus' ship and secretly rebuild an identical replica, the mind revolts at the suggestion that this is somehow the same ship, even though it may have the same structure, location, and components as the original.

These examples illustrate a particular kind of idea that current definitions of complex systems fail to capture: that of continuity through space and time as a central component of identity. The philosopher David Wiggins (1967) uses spatiotemporal contiguity as the defining characteristic of identity. Although this works well in many cases, it requires subtle qualifications to deal with things like spatially discontinuous nation states and spatiotemporally overlapping natural objects. Philosophers, scientists, and sociologists have had difficulty coping with such questions in many different arenas. The same problem arises over species concepts in evolutionary biology. The old definition of species as immutable entities having some essential property or set of properties that could be determined from a single type specimen was gradually transformed as systematists thought through the full implications of Darwin's ideas and realized that species change over time. The key distinction that led to the formulation of the evolutionary and phylogenetic concepts of species was that made by biologist Michael Ghiselin (1966, 1974, 1987) and philosopher David Hull (1974, 1976): species are natural individuals, but not natural kinds. They are not of the same nature as gold or lead, which remain gold and lead and would do so even if it were possible to transform one into the other. Species, like societies, ecosystems, and economies, are mutable, dynamic entities that change over time. On the other hand, unlike species, which are spatially scattered as individuals and populations, ecosystems and societies are typically localized and spatiotemporally contiguous. The lesson from the Ghiselin-Hull approach to species is that mutable, dynamical entities need not have essential properties that define a kind that is present in all of their parts, i.e., some ecosystem essence that can be recognized wherever one is within a specific ecosystem, although that is not ruled out, either. Instead, this approach maintains that identity is determined by dynamical relations among the parts. The problem is to find suitable dynamical relations that determine system identity by binding the system together. These are the sort of natural properties that we should look for, rather than localized properties that are found in every part of the system.

Our goal is not to provide system definitions, which are context-dependent. Rather, we need guidelines that enable us to say unambiguously whether or not our particular ship remains that of Theseus under a wider range of conditions and possible events than our current definition can cope with. A reasonable addition to current definitions would be to recognize that system identity resides in the continued presence, in both space and time, of key components and key relationships. Theseus' ship may move in space and will move in time for as long as it exists; it maintains its identity not only because its planks and sails remain in the appropriate form, but also because the nature of the parts and their relationships to one another have remained the same since Theseus himself first owned the ship. Similarly, although Theseus' ship may move from the harbor to the ocean, it can be traced back in time to its original location. Although this perspective on identity will permit gradual, and not necessarily linear, change from one kind of system to another through a series of intermediate stages, saltationary change will always result in a new system.

An adequate working specification of a complex system should thus encompass the following: (1) the system components, which may be defined in 
varying degrees of detail; (2) the relationships between system components; (3) the location and spatial scale at which the definition is applicable and the importance, or lack thereof, of spatial constancy; and (4) the temporal scale at which the definition is applicable and the author's perspective on the question of identity through time. This final point is essential to the distinctions that we wish to make in the second half of the paper. These four points are logically related and mutually constraining, in the sense that the relationships among system components constrain the types of components that are suitable for maintaining identity. At the same time, the components determine the sort of relations that they can have with each other and still maintain a cohesive system. Unlike a ship, which is a designed artifact, complex systems of the kind that are studied in ecology, sociology, and economics must emerge naturally from the interactions of their components, and their very possibility depends on both the nature and the existence of their components. Furthermore, the notion of a system component itself depends on the mutual constraints of system relations and component nature. Although the atoms making up a complex system are constituents, they are not really components, because they can vary freely, and typically do, without changing the nature of the system. Being a component must be understood in terms of having a relevant role in the overall functioning of the system, not just in being there as a constituent of the system. Last, the scale and limits of the interactions will determine the scale and limits of the system itself, both spatially and temporally, as well as the nature of the boundaries of the system and the ways in which it is nested within larger systems.

Given these points and their consequences, in the second half of this paper we have adopted the view that complex systems are defined by the nature of their main components, the relationships of these components to one another, and the maintenance of both spatial and temporal continuity, i.e., systems may move in space and inevitably move in time, but saltation in either instance constitutes a loss of identity. From this point of view, a complex system is a network of components connected by various dynamical relations that include inputs, outputs, and external constraints. The main problem of system identity or unity is to decide what is internal to the network and what is external. Collier (Collier 1986, 1988, 2003, Collier and Hooker 1999) has suggested in other contexts that the best way to decide dynamical unity is to compare the strength of internal relations among components with those of external relations. This is not always possible, because the relations come in degrees and vary in kind. Furthermore, only some of the relations are relevant to system unity. Which relations are relevant is an empirical question that varies for each type of dynamical system. Different types of complex systems may require different kinds of relations; however, as we suggested in the last paragraph, these relations should have a role in the overall functioning of the system. The closure of such relations determines the dynamical unity of the system.

Bounding complex systems will typically be immensely complex, and simplifications will be needed. For example, Ulanowicz (1986) developed a network account that relies on the strength of flows of carbon, reasoning that carbon flows are a good stand-in for species interactions, although they do not capture behavioral interactions that may be important to ecosystem unity. Nonetheless, he was able to create workable models of trophic relations for complex estuarine ecosystems using this model and devise a measure of connectedness and ecosystem function based on information.

The intricacy of complex system behavior becomes an issue if we are interested in whole system function. Even where specific issues like trophic interactions are studied, highly unpredictable behaviors may occur (e.g., Barkai and McQuaid 1988). Complex dynamical systems are emergent from their components and their relations and cannot be circumscribed by single closed models. Rosen (1991) explains this point in detail, in full logical form, although he (unnecessarily, we think) identifies all such systems with living systems. The emergent properties of complex systems mean that the models that are used will need to be more open than has traditionally been the case, and we will typically need more than one model to capture essential behaviors; even then, we will be unable to obtain a fully circumscribed combination of models. This claim follows from both the nature of complex dynamical systems, especially self-organizing ones, and from some of the problems in logic that are discussed by Gödel and Turing. The choice of which models to use in the study of a complex system then becomes a pragmatic issue rather than a philosophical one: because there is no single "correct" model, it is useful to have a set of different types of models available to use and to guide 
empirical work.

\section{METAMODELS AND SYSTEM CHANGE}

\section{Science and metamodels}

Science is driven very much by how we perceive the world and the mental models that we use to think about observed phenomena. Given the importance of our mental models, which determine the data that we collect, the questions that we consider "interesting," and the ways in which we change our views of the world to accommodate new results, it is perhaps surprising that we do not have a more clear-cut approach to characterizing the essential components of complex systems and the ways in which they interact with one another. The problem is symptomatic of the more general philosophical problem of how best to select discrete, manageable entities to study within a continuous environment.

As we have seen, the reason why there are so few truly general system models is intricately linked to the irreducible complexity of ecosystems, societies, and economies. At the heart of cohesive models of complex systems are a few issues that are extremely difficult to cope with in an empirical investigation. Most complex systems are dynamic entities that span multiple spatial and temporal scales; the distinction between endogenous and exogenous dynamics is not always clear; and, because of their many components, the outcomes of manipulations of the system may differ depending on relatively small differences in starting conditions.

However, despite these complexities there has been some progress toward developing a more general framework for understanding complex systems. The many attempts that have been made to model aspects of different complex systems and an accumulating body of empirical evidence have begun to produce a few more general models that incorporate and summarize the findings of many specific models. Such models are a step back from the immediate process of prediction; they are simple, often tantalizing statements that hint at an underlying order to the workings of the world. Their value comes from the way in which they somehow capture the essential ingredients of many interrelated models in symbolic form. Consequently, we term them "metamodels."

Metamodels are not hypotheses in the commonly used sense. They are not necessarily rigorous quantitative statements, although they must be supported by rigorous quantitative studies. Indeed, they are more a kind of specific metaphor: a way of thinking about things that serves as a powerful tool for the generation of specific hypotheses in specific cases. They are formulations of relationships that are best considered as philosophical statements, akin to Plato's theory of forms. Their value is measured more in terms of their impacts and their usefulness than their immediate scientific testability. Although they have that certain vagueness that is bred of generality, metamodels must be clearly and unambiguously defined. They are not models of specific systems, but at the same time they are not as broad as the "world views" or paradigms outlined by Holling and Gunderson (2002a). Again, although they are are less explicit than the "ecosystem models" discussed by Pickett and Cadenasso (2002), they are considerably less vague than their "ecosystem metaphors." Recognition of the strengths and weaknesses of our own metamodels and consideration of alternative metamodels should serve a useful purpose in refining concepts and highlighting the key distinctions between them.

Holling's adaptive cycle (Holling 1986, 1987, 2001, Holling and Gunderson 2002b) is one of the few well-defined, well-supported interpretations of complex system dynamics. The adaptive cycle is defined by phases that follow one another sequentially (Fig. 1). These can be summarized as resource accumulation ( $r$ to $k$ ), resource release ( $k$ to $\Omega$ followed immediately by system reorganization and reconfiguration $(\Omega$ to $\alpha)$, and re-entry into an accumulation trajectory $(\alpha$ to $k)$. Resource accumulation occurs as the actors in a system do more of what they do. For example, individual trees grow by accumulating nutrients, companies grow by accumulating money, and organizations grow by accumulating members. However, resource accumulation can create vulnerabilities within the system that lead to resource release. Resource release is usually triggered by an external cause or crisis, such as a fire, a market collapse, or a loss of confidence in leadership. During the release phase, accumulated resources are often lost. A period then occurs in which the system reorganizes, possibly changing some of its properties. This is the time during which the system is most open to the introduction of novelty in the form of new species, approaches, or actors. Once sufficient reorganization has occurred for resource accumulation to begin 
again, the cycle resumes. The adaptive cycle is thus a metamodel of a continuous dynamic process in which complex interactions between system components result in a long, slow buildup that contains the seeds of its own subsequent collapse. Other essential ingredients of the metamodel of the adaptive cycle include a focus on the role of endogenous dynamics, a view of systems as continuous entities in both space and time, and an emphasis on periodic reorganization through endogenous or exogeneous drivers.

Fig. 1. Depiction of a system moving through the adaptive cycle. As the system moves from $r$ to $k$, it accumulates capital and grows in size. Following a perturbation (indicated here by the hammer), it undergoes a period of release ( $k$ to $\Omega$ ) and renewal ( $\Omega$ to $\alpha$ ) before entering the $r$ to $k$ trajectory once again. The red and yellow balls indicate alternative stable states of the same system. The labels $r$ and $k$ are derived from the population growth equation, in which $r$ indicates growth rate and $k$ indicates carrying capacity. As the first and last letters of the Greek alphabet, respectively, $\Omega$ and $\alpha$ signify ending and beginning.

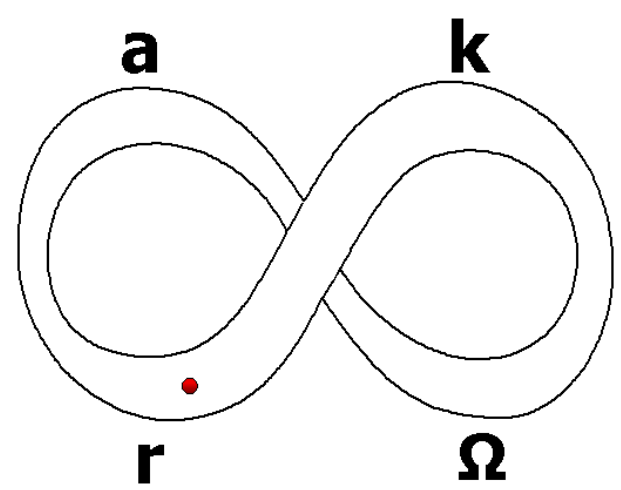

View animated version of this figure
The behavior of systems of a certain kind has been shown to closely match the adaptive cycle. The dynamic between forests, fire, and succession provides a classic example (Fig. 2). Because it seems to fit many ecological and social systems and few or no counter-examples have been described, the adaptive cycle has been criticized for being too broad. Few critics have appreciated that the adaptive cycle is really a metamodel, i.e., a broader class of model that encapsulates the key dynamics of numerous other models. In this section, we argue that, first, there are other metamodels of system function and, second, these metamodels should not be expected to synthesize across the same system dynamics as the adaptive cycle, because they are models of a fundamentally different kind of system. Evaluation of the adaptive cycle has yet to move beyond systems or models that have essentially the same dynamics as the models from which the metamodel was constucted; from this comes the illusion that the adaptive cycle explains everything. By rigorously defining the properties that are expected of systems that match different kinds of metamodels, we can move a step closer to understanding what the central ingredients of particular system behaviors are and develop an improved appreciation of their commonalities and differences.

Although the adaptive cycle offers a persuasive approach to characterizing and understanding system dynamics, it is only one of a set of possible metamodels that might explain or clarify different aspects of system behavior. We propose that further attempts to develop, refine, and examine alternative metamodels will help us to make further progress in studies of complex systems. To find exceptions that do not follow adaptive cycle-type dynamics, we must look for systems that are discontinuous, that exhibit few or no relevant internal dynamics or are continuously overwhelmed by external forces, and that have little or no self-organizational ability or "adaptive capacity." In the next section, we consider some candidates for alternative metamodels that may explain different kinds of complex system phenomena. Some of the most interesting alternative metamodels for complex systems may be those that mirror many of the dynamics of the adaptive cycle but can be distinguished from it in one or more crucial ways. 
Fig. 2. The adaptive cycle illustrated for a forest ecosystem. Carbon accumulates as the pine forest grows $(r$ to $k$ ). Carbon and other nutrients are released by fire ( $k$ to $\Omega$ ), and remain largely absent for a short period $(\Omega$ to $\alpha)$. At $\alpha$, a range of propagules enters the system from seed banks and surrounding areas; successional processes ensue, influenced by the abiotic environment; and a pine forest re-establishes itself. In reality, this process will seldom occur for an entire forest, meaning that the "new" forest carries with it elements of the old.

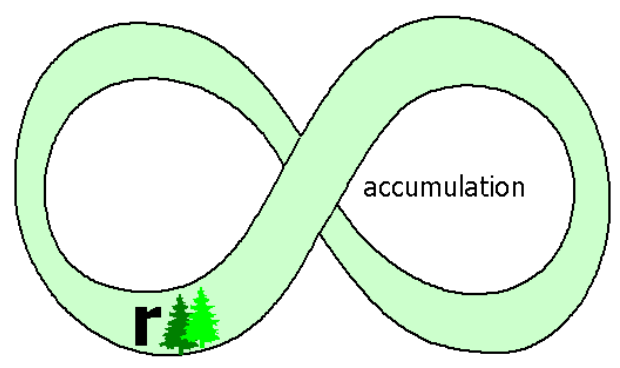

View animated version of this figure

\section{Alternative metamodels}

Alternative metamodels will be relevant wherever a system is in clear violation of one of the central features of the adaptive cycle. We use a strict definition of the adaptive cycle, believing that it is only through making the details of each metamodel clear and explicit that we will be able to progress toward a consistent philosophical framework. Continuous modification of the adaptive cycle to encapsulate all possible complex system dynamics is neither useful nor desirable. It is also important to note that, because system definitions are applied at particular spatiotemporal scales, the nature of system dynamics may differ between scales. It is likely that many systems may obey one metamodel at one scale and another metamodel at a broader scale.

\section{Random walk}

The most obvious alternative metamodel is encapsulated in unpredictability. Under this model, complex systems wander randomly through a multivariate space. Their dynamics and components undergo stochastic changes at irregular intervals of time. There is no cycling and no particular regularity in system properties. This model is primarily a "straw man" that exists to be disproven and has been disproven in many cases. Nonetheless, it is worth stating explicitly because it is a null model against which other models must be contrasted; alternative metamodels must encapsulate some form of order or repetition. A topical example of a largely stochastic ecological process is that of the location and timing of species invasions (May 1976).

\section{Replacement}

The adaptive cycle is not an appropriate metamodel for systems that lose their continuous identity in either space or time. Such systems may follow after one another, be similar to one another, and occur in the same location as one another, but they are not true examples of a single system that undergoes a periodic cycle of growth and reorganization. An equivalent situation would be one in which an orphan was repeatedly adopted by short-lived foster parents. Although the child as a passionate observer might experience emotions akin to the adaptive cycle in each instance and each couple would consist of a father and a mother, successive parents could not reasonably be considered the same. Replacement may occur with a predictable or semipredictable frequency and may be weakly reinforced by internal dynamics. These characteristics make the replacement metamodel distinct from a purely stochastic metamodel.

There are many examples of social systems replacing one another. As particular social groups have grown powerful and invaded their neighbors, a process of assimilation of the martially weaker culture into the stronger culture has often occurred. In many cases, such assimilation has resulted in a loss of key aspects of the identity of the conquered society, including such things as language, artifacts, and genetic uniqueness. This situation conforms to the replacement metamodel.

An example of a biological system that fits a replacement metamodel better than it fits an adaptive cycle metamodel is that of a lotic (flowing 
water) ecosystem. The quantity of water flowing in a stream is largely an exogeneous property of the system. Following a severe flood, sediments are rearranged and many organisms are swept away. The community that remains or is reconstituted after the disturbance is a combination of legacies or "ecological memory" from the previous community, plus new colonizers. Similarly, volcanoes on oceanic islands can result in the replacement of entire ecosystems, with substantial changes to both abiotic and biotic components (Fig. 3). In both of these examples, there may be profound changes in the components from which the system is constructed and their relationships to one another. According to the continuity criterion, what remains is a different system. There is no fundamental dynamic of reorganization, no return to the previous trajectory, and not necessarily an obvious accumulation of "capital," in the sense that forests accumulate wood or companies accumulate money, between disturbance events. Although the system is dynamic, the adaptive cycle does not offer an adequate summary of it. Obviously, at smaller or larger scales, alternative kinds of system dynamics, including the adaptive cycle, may be possible.

The stream example also illustrates the role of subjective choice in our definitions of study systems. For some purposes, the physical environment might be the best focus for study, independent of changes in biota after a flood. If the physical location places sufficiently large constraints on the kind of ecosystem that can exist in that location, one could say that, because the physical system is preserved, the ecosystem is preserved. However, if the relations among the biota introduce important constraints on the functional properties of the system, e.g., the processing of allochthonous particulate organic matter, and the functional properties of the system change after a flood, then the strongest claim that can be made may be merely that the physical environment places important constraints on successive ecosystems. By forcing researchers to pay attention to the overall properties that define their systems, consideration of system identity in the context of multiple alternative metamodels can help researchers to identify their own particular biases and avoid too narrow a focus on the problems that they are interested in.
Fig. 3. A simple depiction of ecosystem replacement on an oceanic island such as Krakatoa. The volcano changes the topography of the island and covers it in ash and lava, creating a different abiotic environment. Recolonization occurs at random from bird-dispersed seeds that differ substantially from those of the vegetation that was there prior to the volcano. Spatiotemporal continuity of the ecosystem is completely lost in this example.

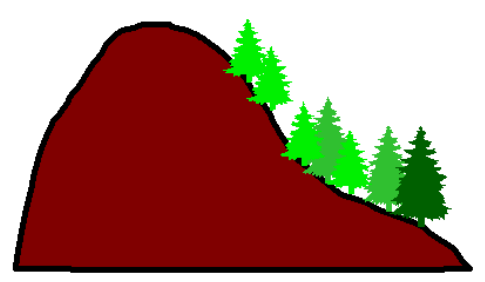

View animated version of this figure

Systems in which substantial legacies are left after disturbances fall into a gray area between replacement and reorganization. The ends of the continuum, i.e., disturbances leave no legacy or disturbances leave a legacy of the entire system, are easy to classify as instances of replacement or the adaptive cycle, respectively. At locations mid-way between these two extremes, there is no simple answer. In an ecosystem, the solution will depend on the proportion of the subsequent biotic community that is endogenous, the extent to which the abiotic environment was altered by the disturbance, and the degree to which biotic interactions in the new ecosystem have changed. The concept of path dependency (Rosen 1991), in which trivial-seeming "accidents" or initial conditions can have persistent effects on the subsequent trajectory of the system, is also relevant here. A system's future identity may be as much the result of a chance event, such as faulty voting machines in one county in Florida, as of any kind 
of predictable self-organizing or environmentally forced process.

\section{Succession}

The adaptive cycle uses the older metamodel of ecological succession as its fundamental dynamic. Successional theories are also common in other disciplines, for example, in the ideas that human societal organization changes as population size increases, that the basis of a typical economy tends to shift from hunting to farming to industry, or that companies need to employ more staff as they accumulate capital. Holling's important insight into the successional process was to recognize the time of reorganization that occurs between successional events as an integral part of complex systems and to make it explicit. Any system that does not undergo both succession and a subsequent reorganization phase of some kind does not fit the adaptive cycle metamodel.

As a thought experiment, imagine that, through careful management, a system could be kept in the " $r$ to $k$ " or accumulation phase of the adaptive cycle indefinitely. Next, imagine that the manager could gradually remove his or her influence by developing the self-organizational capacity of the system. Finally, imagine that the manager could completely withdraw and leave the system perpetually stuck in the $r$ to $k$ phase. To argue that this situation is only possible by the maintenance of adaptive cycles at a smaller scale is to miss the point. The point is that such a system, if it existed, would fit the successional metamodel better than it does the adaptive cycle. Although decades of work have shown that few or no real-world systems fall into this category (Holling and Meffe 1996), without these rigorous tests of real-world dynamics we would not be able to dismiss the successional metamodel so readily.

\section{Dynamic limitation}

Another potential metamodel is encapsulated in the idea that complex systems are constrained by external drivers. The process of dynamic limitation can be visualized as a kind of "jack-in-the-box," in which complex systems are constantly pushing against external limits. As the system boundaries change along any of the multiple axes that pertain, e.g., in an ecosystem such limits might occur in space, substrate, or temperature, components of the system either go extinct or expand to exploit the full plausible state space. This metamodel is distinct from the adaptive cycle. There is no accumulation or reorganization, and cycling is not a necessary condition; limitation comprises a set of forward and backward movements as if between two dance partners, with an occasional "explosion" or release when constraints are removed.

The process of dynamic limitation is distinct from the replacement model. The internal dynamics of the system will depend heavily on system processes, and there is no reason why the endogenous or finergrained exogenous dynamics should not follow the adaptive cycle metamodel, but the dynamic limitation model is applied at a broader scale than this. Dynamic limitation is primarily a boundary condition, not a system-wide driver. Changes in limitation do not produce an entirely new system, and there is no obvious replacement event, except possibly through some kind of accumulation of small changes. In this metamodel, exogenous drivers "tinker" with some of the pieces of the system, and endogenous variation occurs at such a fine scale that it is largely irrelevant. The dynamic limitation metamodel does not permit alternate stable states, although these may obviously arise through other mechanisms, because it does not incorporate fundamental shifts in system controls.

Limitation describes many chaotic systems as well as those that slowly expand and contract along a shared boundary. For example, over very long time periods, the adaptive cycle-type dynamics of boreal forests may be irrelevant to their persistence. Glaciers grow or recede as the climate changes, and the location of the forest line moves north or south (Fig. 4). Trees recolonize areas that are left free of ice and die when they are covered. At the scale of analysis, there are no accumulation and release of resources and few alternative trajectories, simply a slow back-and-forth movement that may, if the glaciers advance too far, result in the loss of one of the interacting components. 
Fig. 4. A simple depiction of limitation at an iceforest boundary. At the time scale of interest, the boundaries of each system simply move back and forth with no evidence of cycling.

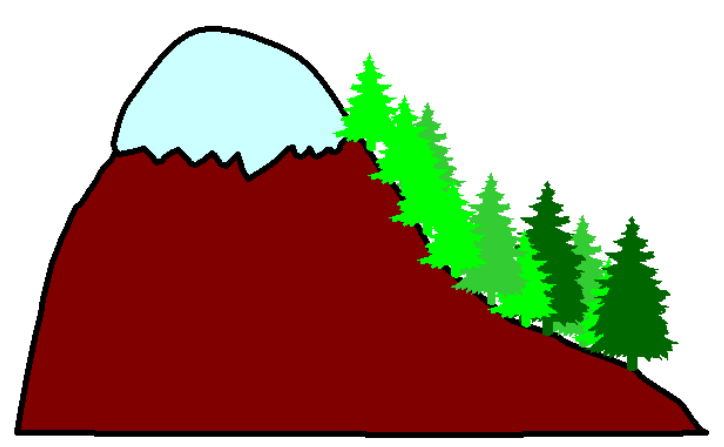

View animated version of this figure

\section{System evolution}

The theory of evolution provides us with another example of a metamodel and, like the adaptive cycle, has been criticized for not being falsifiable. Holling and Gunderson (2002b) incorporate "nature resilient" within a world view of "nature evolving," suggesting perhaps that they see the adaptive cycle as one member of a subset of evolutionary metamodels. Evolution is undeniably important as a mechanism by which species assemblages arise, and it plays a key role in self-organization within complex systems that have an ecological component. However, we do not regard it as an appropriate metamodel for the kinds of general, whole-system dynamics that we are discussing here. In the strict sense, it is not obvious that all complex systems can be said to evolve. Organisms differ from most commonly considered complex systems such as societies or ecosystems in that they all carry a complete blueprint for future copies of themselves. Darwinian evolution assumes a mechanism by which variations in competing complex systems are generated and selection removes individuals that are poorly suited to current conditions. Although there may be complex system parallels to anagenesis or gradual speciation with no divergence, cladogenesis (branching) at an ecosystem level would be difficult to demonstrate. Applying the assumptions of a rigid evolutionary metamodel of adaptation to entire ecosystems leads inevitably to the murky arena of group selection. Because many ecosystems are unique and, aside from anthropogenic impacts, there is little opportunity for one ecosystem to displace another, it seems that the evolutionary metamodel is not entirely appropriate for the kinds of complex systems that we are interested in. Rather than dilute the clear insights of Darwin's theory by applying it outside its original context, we consider it wiser to capture change in entire ecosystems using other conceptual frameworks.

A stronger case for evolutionary change may exist for societies, which do not necessarily incorporate their abiotic environment in the way that ecosystems do, and a common form of cladogenesis occurs when a subset of a human population migrates. For example, the offspring of the Maori people developed distinct cultures on several different islands. However, cultural evolution is not evolution in the sense that is accepted by biologists. Although Dawkins (1976) has proposed that cultural units or "memes" similar to genes exist, cultural evolution proceeds in a Lamarckian fashion, i.e., by the inheritance of acquired characteristics. Despite their superficial similarities, the underlying mechanisms that drive cultural variation and the generation of new ideas are generally very different from those that drive genetic and phenotypic variation. It is reasonable to argue that accumulated cultural changes would eventually lead to a new society or a new economy. However, because such changes occur through time and in space, they would either be consistent with our criteria for the maintenance of spatiotemporal identity, as in the evolutionary species concept, or would fit one of the metamodels that have already been proposed.

\section{DISCUSSION}

The different metamodels that may be applicable to individual complex systems are summarized in Table 1. This list is not intended to be exhaustive; we have simply tried to demonstrate some plausible alternatives to the adaptive cycle metamodel.

In each instance, the scale of the definition is relative 
Table 1. A provisional categorization of metamodels according to their defining characteristics. They can be distinguished on the basis of whether the system maintains a continuous identity through time; whether alternative stable states (implying some form of self-reinforcement) are possible in the same physical location; and whether the role of exogenous variables is strong or weak. As explained previously, the evolution model is excluded because we do not regard it as an appropriate whole-system model for ecosystems, societies, and economies.

\begin{tabular}{llll}
\hline \hline Metamodel & $\begin{array}{l}\text { Continuous } \\
\text { identity }\end{array}$ & $\begin{array}{l}\text { Alternative stable } \\
\text { states possible }\end{array}$ & $\begin{array}{l}\text { Exogenous } \\
\text { forcing }\end{array}$ \\
\hline Random walk & No & No & Yes \\
Replacement & No & Yes & Yes \\
Limitation & Yes & No & Yes \\
Succession & Yes & No & No \\
Adaptive cycle & Yes & Yes & No/yes \\
\hline
\end{tabular}

to the scale at which the system boundaries have been set. Endogenous drivers are contained within the system and include such processes as predation or assimilation, competition, facilitation, and exchanges of information. Exogenous drivers are external to the system and typically include energy inputs and disturbances, many of which, such as flooding or market fluctuations, have their origins in processes that occur at a broader spatial or temporal scale. Depending on the scale of analysis, different drivers may be endogenous in some instances and exogenous in others. It is possible that there is a predictable shift from certain kinds of metamodel to others as the scale of analysis increases. Levin (1999) considers that prediction is generally easier at coarser scales of analysis, suggesting that metamodels may be more readily applied at certain scales. It remains to be seen whether the system dynamics that we summarize in metamodels are predictable from aggregations of smaller units, or if they only emerge at certain scales or as a global consequence of local optimality or local adaptation (Levin 1999).

At a given scale or scales of analysis, system continuity or maintenance of identity occurs when successive stages in system development grade into one another with no spatial or temporal breaks. A loss of identity occurs when there is spatial or temporal separation of a system from its predecessor, where such exists. Again, the question of what constitutes a loss of identity is defined according to the scale of analysis and the potential for variation in the system. For example, colonization of an entirely bare piece of land by plant propagules from adjacent habitats defined as being outside the system at the scale of analysis would indicate a new identity, but the same event might indicate system continuity if the study system is defined at a broader spatial scale.

The metamodels of system dynamics that we have discussed are not in competition with the adaptive cycle as a "theory of everything." Nor are they necessarily exclusive of one another, even for the same system. Each can be correct; each has limited applicability. Their value is in helping us to recognize certain system attributes that are relevant to the question that we are interested in, and particularly in understanding the dynamic relationships between system components. Metamodels describe a certain kind of dynamic that may or may not apply in a specific case. For a metamodel to be useful, it has to fit at least one real-world case and be inappropriate in at least one other case. The existence of alternative metamodels implies that not all systems fit the adaptive cycle as it is currently formulated.

The adaptive cycle itself may need to be further subdivided to recognize distinctions between qualitatively different system dynamics. Some systems undergo regular, predictable cycles that continually return to a single state. In other systems, 
changes are gradual and system identity is maintained, but alternate stable states are in evidence. We can envisage many instances in which the distinction between these two different kinds of system dynamics could be important. For example, they could be critical where the continuous provision of some ecosystem service is essential for human well-being. If forests in one area undergo predictable cycles of fire and budworm outbreaks, their management becomes a fundamentally different problem from forests in another area that may gradually give way to grasslands or dense thickets of shrubs. The question of whether such a state change indicates adaptive cycling or replacement is a difficult one that can only be answered by the consideration of long-term dynamics.

One of the central problems in coping with the analysis and management of complex systems is precisely that they are complex systems. Even at this refined philosophical level, there are many difficulties in applying metamodels across multiple scales. Metamodels are not as explicit as hypotheses and are more general than models, but they are also more rigid than metaphors or world views. They can be rejected only on the grounds of inconsistency, $i$. e. through rational argument, and can be discarded only if it can be demonstrated that they have no foundation in the real world. A self-evident but frequently ignored property of metamodels is that we cannot explore their limitations by considering only the types of systems that gave rise to the metamodel in the first place. Metamodels must be defined clearly and rigidly if they are to be useful, so that they can be contrasted against alternatives that may be similar in many respects. At the same time, however, it must be remembered that viewing complex systems in different ways does not make them distinct. The abstract attributes that we describe with metamodels are, in a sense, abstract and coarse-grained properties that we might expect any ecosystem to display in some way or another.

An interesting implication of matching real-world systems to metamodels is that systems that are best explained by fundamentally different metamodels may require very different kinds of management strategies. Although these differences seem trivial in some instances, they may offer some extremely useful insights in others. For example, systems that obey a replacement dynamic may exhibit superficially similar behavior to systems with an adaptive cycle dynamic, but in reality may have much larger attendant uncertainties and a much greater range of possible trajectories. Systems that behave according to different metamodels may have fundamentally different levels of resilience and vulnerability, an area that would merit further research.

Responses to this article can be read online at:

http://www.ecologyandsociety.org/vollo/iss 1/art29/responses/

\section{Acknowledgments:}

We are grateful to the many people who have debated these and related issues with us. Ben Bolker first suggested the example of glaciation to $G C$, and Gene Likens challenged $G C$ on the definitions of ecosystems and landscapes. At various stages during the development of this manuscript, useful comments were provided by Resilience Alliance members; we particularly thank Buzz. Holling, Steve Carpenter, David Cumming, Jon Norberg, and Marten Scheffer for their feedback on earlier drafts. This is Florida Agricultural Experiment Station Journal Series No. R-10603.

\section{LITERATURE CITED}

Barkai, A., and C. McQuaid. 1988. Predator-prey inversion in a marine benthic ecosystem. Science 242:62-65.

Clough, A. H., editor. 1992. Plutarch: lives of noble Grecians and Romans. Modern Library Series. Translated by John Dryden. Modern Library, New York, New York, USA.

Collier, J. 1986. Entropy in evolution. Biology and Philosophy 1:5-24.

Collier, J. 1988. Supervenience and reduction in biological hierarchies. Philosophy and Biology. Canadian Journal of Philosophy Supplementary Volume 14:209-234.

Collier, J. 2003. Hierarchical dynamical information systems, with a focus on biology. Entropy 5:100-124. 
Collier, J., and C. A. Hooker. 1999. Complexly organised dynamical systems. Open Systems and Information Dynamics 6:241-302.

Dawkins, R. 1976. The selfish gene. Oxford University Press, Oxford, UK.

Ghiselin, M. T. 1966. On psychologism in the logic of taxonomic controversies. Systematic Zoology 15:207-215.

Ghiselin, M. T. 1974. A radical solution to the species problem. Systematic Zoology 23:536-544.

Ghiselin, M. T. 1987. Species concepts, individuality, and objectivity. Biology and Philosophy 2:127-144.

Gunderson, L. H., and C. S. Holling, editors. 2002. Panarchy: understanding transformations in human and natural systems. Island Press, Washington, D.C., USA.

Holling, C. S. 1986. The resilience of terrestrial ecosystems; local surprise and global change. Pages 292-317 in W. C. Clark and R. E. Munn, editors. Sustainable development of the biosphere. Cambridge University Press, Cambridge, UK.

Holling, C. S. 1987. Simplifying the complex: the paradigms of ecological function and structure. European Journal of Operational Research 30:139-146. Republished 1995 in Futures 26:598-609.

Holling, C.S. 2001. Understanding the complexity of economic, social and ecological systems. Ecosystems 4:390-405.

Holling, C. S., and L. H. Gunderson. 2002a. In quest of a theory of adaptive change. Pages 3-22 in L. H. Gunderson and C. S. Holling, editors. Panarchy: understanding transformations in human and natural systems. Island Press, Washington, D. C., USA.

Holling, C. S., and L. H. Gunderson. $2002 b$. Resilience and adaptive cycles. Pages 25-62 in L. H. Gunderson and C. S. Holling, editors. Panarchy: understanding transformations in human and natural systems. Island Press, Washington, D.C., USA.

Holling, C. S., and G. K. Meffe. 1996. Command and control and the pathology of natural resource management. Conservation Biology 10:328-337.

Hull, D. L. 1976. Are species really individuals? Systematic Zoology 25:174-191.

Hull, D. L. 1978. A matter of individuality. Philosophy of Science 45:335-360.

Levin, S. 1999. Fragile dominion: complexity and the commons. Perseus, Cambridge, UK.

Likens, G. E. 1992. The ecosystem approach: its use and abuse. Excellence in Ecology, Book 3. Ecology Institute, Oldendorf/Luhe, Germany.

May, R. M. 1976. Simple mathematical models with very complicated dynamics. Nature 261:459-467.

Odum, H. T. 1971. Environment, power, and society. Wiley, New York, New York, USA.

O'Neill, R. V. 2001. Is it time to bury the ecosystem concept? (With full military honors of course!). Ecology 82:3275-3284.

Pickett, S. T. A., and M. L. Cadenasso. 2002. The ecosystem as a multidimensional concept: meaning, model, and metaphor. Ecosystems 5:1-10.

Rosen, R. 1991. Life itself. Columbia University Press, New York, New York, USA.

Sagoff, M. 2003. The plaza and the pendulum: two concepts of ecosystem science. Biology and Philosophy 18:529-552.

Tansley, A. G. 1935. The use and abuse of vegetational concepts and terms. Ecology 16:284-307.

Wiggins, D. 1967. Identity and spatio-temporal continuity. Oxford University Press, Oxford, UK.

Ulanowicz, R. E. 1986. Growth and development: ecosystems phenomenology. Springer, New York, New York, USA. 